Revue d'histoire de l'Amérique française

REVUE D.HISTOIRE DE L'AMÉRIQUE FRANÇAISE

\title{
Les revues littéraires au Québec comme réseaux d'écrivains et instance de consécration littéraire (1840-1870)
}

\section{Maurice Lemire}

Volume 47, numéro 4, printemps 1994

URI : https://id.erudit.org/iderudit/305278ar

DOI : https://doi.org/10.7202/305278ar

Aller au sommaire du numéro

Éditeur(s)

Institut d'histoire de l'Amérique française

ISSN

0035-2357 (imprimé)

1492-1383 (numérique)

Découvrir la revue

Citer cet article

Lemire, M. (1994). Les revues littéraires au Québec comme réseaux d'écrivains et instance de consécration littéraire (1840-1870). Revue d'histoire de l'Amérique française, 47(4), 521-550. https://doi.org/10.7202/305278ar
Résumé de l'article

Les revues littéraires sont le plus souvent des organes de cercles littéraires plus ou moins officieux. Elles jouent un double rôle; elles rassemblent des écrivains qui partagent certaines visions et elles contribuent à former des réputations en consacrant des oeuvres. Dans une littérature en voie de formation, elles servent également de stade intermédiaire entre le journal et le livre. C'est dans cet esprit que nous nous sommes penché sur des répertoires et des périodiques comme Le répertoire national, $L a$ Ruche littéraire, $L$ 'Écho du Cabinet de lecture paroissial, le Journal de l'Instruction publique, Les Soirées canadiennes, Le Foyer canadien et la Revue canadienne pour découvrir quels en étaient les principaux animateurs et collaborateurs et quelle était leur politique éditoriale. De cet examen, il ressort que c'est à l'intérieur du cadre des revues que se profilent les premiers mouvements littéraires.
Tous droits réservés @ Institut d'histoire de l'Amérique française, 1994

Ce document est protégé par la loi sur le droit d'auteur. L'utilisation des services d'Érudit (y compris la reproduction) est assujettie à sa politique d'utilisation que vous pouvez consulter en ligne.

https://apropos.erudit.org/fr/usagers/politique-dutilisation/ 


\title{
LES REVUES LITTÉRAIRES AU QUÉBEC COMME RÉSEAUX D'ÉCRIVAINS ET INSTANCE DE CONSÉCRATION LITTÉRAIRE
} (1840-1870)

\author{
MAURICE LEMIRE \\ Centre de recherche en littérature québécoise (CRELIQ) \\ Université Laval
}

\begin{abstract}
RÉSUMÉ
Les revues littéraires sont le plus souvent des organes de cercles littéraires plus ou moins officieux. Elles jouent un double rôle; elles rassemblent des écrivains qui partagent certaines visions et elles contribuent à former des réputations en consacrant des ouvres. Dans une littérature en voie de formation, elles servent également de stade intermédiaire entre le journal et le livre. C'est dans cet esprit que nous nous sommes penché sur des répertoires et des périodiques comme Le répertoire national, La Ruche littéraire, L'Écho du Cabinet de lecture paroissial, le Journal de l'Instruction publique, Les Soirées canadiennes, Le Foyer canadien et la Revue canadienne pour découvrir quels en étaient les principaux animateurs et collaborateurs et quelle était leur politique éditoriale. De cet examen, il ressort que c'est à l'intérieur du cadre des revues que se profilent les premiers mouvements littéraires.
\end{abstract}

\begin{abstract}
Literary reviews are often seen as the more or less official mouthpieces of early literary societies. As such, they play a double role: first, they bring together authors with a common purpose of vision and secondly, they contribute to the reputations of these authors by promoting their works. In the case of a nascent literature, or one which is still in its early stages of development, these reviews also act as an intermediary form of publication, which lies somewhere between the newspaper article and the book. With this idea in mind, the author of this paper undertook to examine a certain number of literary reviews (and one anthology, Le répertoire national): La Ruche littéraire, L'Écho du Cabinet de lecture paroissial, le Journal de l'Instruction publique, Les Soirées canadiennes, Le Foyer canadien, and the Revue canadienne. These serial publications were scrutinized with a view to discovering their main contributors and their editorial practices over a period of time. From this study, it appears that reviews such as these are in large part responsible for creating the earliest literary movements in French Canada.
\end{abstract}


Certains historiens situent les débuts de la littérature québécoise avec la parution du recueil de poésie de Michel Bibaud en 1830 et du roman du jeune Philippe Aubert de Gaspé en 1837, comme si le livre était le support unique de la littérature. Cette vision plutôt arbitraire laisse croire que ces œuvres apparaissent comme des oasis en plein désert, alors que les journaux de la même époque publient des poèmes, des contes, des discours et des conférences. L'appareil éditorial dont peut se doter une société correspond à l'état de son public-lecteur. Ce n'est en fait qu'à partir du moment où l'instruction se généralise que l'on voit surgir une industrie du livre telle que nous la connaissons maintenant. Mais avant d'en arriver là, l'imprimé a passé par des stades intermédiaires. Pour les Canadiens, la presse constitue le premier véhicule de la littérature. Depuis son avènement, elle ne cesse de présenter des poèmes, des nouvelles et des bluettes la plupart du temps sans signature. Bien qu'elle ait été longtemps la seule possible, cette formule place cependant la littérature sur le même pied que les autres écrits sans distinguer le littéraire du non-littéraire. Aussi fragiles que leur support, ces textes tombent dans l'oubli dès leur parution sans contribuer ni à enrichir le corpus ni à établir des réputations.

$\mathrm{Au}$ cours des années 1840, la situation change: les maisons d'éducation, les journaux et surtout des associations se multiplient. Un lectorat plus nombreux et des regroupements moins éphémères permettent de mettre au point un système éditorial à mi-chemin entre le journal et le livre. La formule reste encore indéterminée: ce n'est pas la revue proprement dite, car à l'exception de La Revue canadienne de 1864 , on préfère, dans certains cas, le sous-titre de «recueil de littérature», qui tient plus de l'anthologie que de la revue. Des périodiques comme Le Ménestrel, l'Album littéraire et musical de la Revue canadienne et l'Album littéraire et musical de La Minerve n'ont rien de commun avec les revues, mais ils se distinguent des journaux en ce qu'ils s'adressent à un public particulier. Ces publications ont donc en commun de regrouper des collaborateurs à dominante littéraire et de définir un lectorat spécifique.

Ces premiers albums marquent un progrès dans la détermination du public que caractérise une certaine culture musicale et littéraire. L'introduction du piano dans les salons démarque nettement le divertissement savant fondé sur une culture musicale et littéraire du divertissement populaire. C'est pour ce public que l'on publie des feuilletons comme La Fille du brigand, Charles Guérin et Une de perdue, deux de trouvées... Mais ces publications n'ont pas comme objectif immédiat de promouvoir la littérature.

Au contraire, Le répertoire national se présente comme un recueil qui fait le point périodiquement sur l'état de la littérature. Plusieurs 
suppléments seront publiés au cours de la seconde moitié du siècle. $L a$ Ruche littéraire et politique, dont le premier numéro date de 1853 et le dernier, de 1859, se veut exclusivement littéraire, même si son rédacteur, Henri-Émile Chevalier, est tenté à l'occasion par la politique. Revue consacrée avant tout à la pédagogie, le Journal de l'instruction publique s'adresse aux instituteurs, mais accorde, sous la direction de P.-J.-O. Chauveau, une attention soutenue à la littérature. Il en va de même pour L'Écho du Cabinet de lecture paroissial qui veut combattre les mauvaises lectures en encourageant les bonnes. Ce sont surtout les grandes revues des années 1860 qui témoignent de la formation d'un milieu littéraire canadien. Mais la disparition des Soirées canadiennes et du Foyer canadien, après à peine six ans d'existence, rappelle aux éditeurs que les vrais lettrés ne sont peut-être pas encore assez nombreux pour faire vivre des revues exclusivement littéraires. La Revue canadienne qui s'inspire de la formule de $\mathrm{La}$ Revue des Deux Mondes a pour elle les promesses de l'avenir parce qu'elle allie à la diversité des intérêts une orientation littéraire précise.

\section{LE RÉPERTOIRE NATIONAL}

Le répertoire national ou Recueil de littérature canadienne n'est pas à proprement parler une revue, bien qu'il ait été publié par fascicules sur une période de deux ans. Dans sa conception, il représente un cas sans précédent dans le monde des lettres. James Huston, un autodidacte formé dans les ateliers de Napoléon Aubin, lui en a certainement emprunté l'idée. En effet le rédacteur du Fantasque avait préconisé dès 1838 de recueillir en volume les textes littéraires épars dans les journaux. Nommé traducteur à la Chambre d'assemblée en 1846, Huston trouve à Montréal un milieu propice à son projet.

Par la publication de son Histoire du Canada en 1845, FrançoisXavier Garneau avait réfuté la première partie de l'assertion de Durham: «Peuple sans histoire et sans littérature». Restait à démentir la seconde. La tâche paraissait à première vue plus facile parce qu'elle se limitait au dépouillement des journaux. Mais l'opération revêtait un caractère plutôt symbolique car elle amorçait le discours constitutif de la littérature canadienne; elle visait implicitement à séparer le littéraire du non-littéraire et, en même temps, à en délimiter les frontières. De plus, elle donnait l'occasion d'identifier les auteurs pour la plupart encore anonymes et de comparer leurs œuvres.

Huston ne pouvait s'acquitter seul de cette tâche. La récupération de la plupart des textes littéraires disséminés dans les journaux depuis la fondation de La Gazette de Québec dépassait les capacités d'un chercheur isolé. Il faut croire qu'il profite d'une conjoncture très 
favorable. Quand il emménage à Montréal, en 1846, la nouvelle capitale du Canada-Uni regroupe alors la plupart des sommités littéraires: Jacques Viger, archéologue réputé, Georges-Barthélemi Faribault, bibliothécaire du Parlement, Étienne Parent, greffier du Conseil législatif, Denis-Benjamin Viger, conseiller exécutif, JosephGuillaume Barthe, rédacteur de L'Aurore des Canadas... De plus, le traducteur s'implique rapidement dans l'Institut canadien, qui rassemble des jeunes intellectuels avides de faire leur marque dans la carrière littéraire, comme Antoine Gérin-Lajoie, Joseph Lenoir, P.-J.O. Chauveau, nouvellement élu député. Il a également ses entrées à la Société des amis grâce à Guillaume Lévesque, traducteur à la Chambre, où il rencontre Louis-Octave Le Tourneux, Charles Lévesque et Louis-Auguste Olivier. C'est encore à Montréal qu'il connaît Charles Laberge, Joseph Papin et la plupart des «treize» qui fonderont bientôt L'Avenir.

Quelle ampleur prend vraiment la cueillette des textes littéraires dans les journaux? La répartition de la matière en quatre volumes aide à apprécier la recherche à effectuer pour produire Le Répertoire. Le premier volume regroupe des textes qui s'échelonnent de 1734 à 1837, tandis que les trois autres portent uniquement sur ceux de la décennie 1838-1848. Autrement dit, plus des trois quarts d'entre eux proviennent d'auteurs encore vivants avec lesquels le compilateur peut communiquer. Même pour le premier volume, il peut certainement consulter Jacques Viger et Michel Bibaud, qui disposent d'une riche documentation sur la période antérieure à 1838 , comme le prouve la place faite aux écrits de Joseph Quesnel et de Joseph Mermet, déposés dans Ma Saberdache. C'est également de là qu'il exhume les premiers poèmes de Denis-Benjamin Viger. Quant à l'œuvre poétique de Michel Bibaud, elle jouit de la seule publication en recueil. FrançoisXavier Garneau, que le compilateur connaît de longue date, se fait un plaisir de lui communiquer ses poèmes, quitte à les remanier pour l'occasion. En effet, la version du Répertoire national diffère de celle des journaux. Pour la production des années 1836, 1837 et 1838, Huston puise abondamment dans Le Populaire dont Leblanc Marconnay a voulu faire un tremplin pour les jeunes auteurs canadiens. Il obtient aussi de la famille les lettres inédites de Chevalier de Lorimier.

La cueillette pour la période d'avant 1838 aurait cependant pu être beaucoup plus considérable si le compilateur avait dépouillé systématiquement les journaux, comme la collection déposée à la Bibliothèque du Parlement lui en offrait la possibilité. Une simple comparaison avec Les textes poétiques du Canada français dont nous disposons maintenant suffirait à nous en convaincre. 
Le problème de Huston est donc moins de recueillir les textes que de les sélectionner. Comme il ne pouvait pas tout inclure, il devait choisir, mais en vertu de quels critères? Il en indique quelques-uns dans sa préface, tout en précisant le sens de sa démarche: n'ambitionnant pas de produire une simple anthologie où seule la valeur littéraire compterait, il présente un corpus qui retrace l'évolution d'une production littéraire pour que les futurs chercheurs puissent en «étudier l'enfance, les progrès et l'avenir». Les textes seraient donc sélectionnés dans le dessein d'illustrer une progression, les premiers servant à faire valoir les derniers. La trajectoire ainsi tracée amènerait le lecteur à la conclusion que la littérature canadienne s'engage dans un processus d'autonomisation qui la consacre comme objet d'étude:

Le lecteur se réjouira, comme nous, en arrivant à l'époque actuelle, de voir combien la littérature canadienne s'émancipe du joug étranger; de voir combien les écrivains, mûris par l'âge et par l'étude, diffèrent en force, en vigueur, en originalité, des premiers écrivains canadiens; de les voir s'élever au-dessus des frivolités et des passions politiques, pour aller à la recherche de tout ce qui peut être vraiment utile au peuple, de tout ce qui peut consolider et faire briller notre nationalitéé

Première règle de sélection: exclusion de tous les écrits politiques. Cette décision, plus accidentelle qu'essentielle à la définition du littéraire, cherche à éviter que Le Répertoire ne soit perçu comme une autre forme de propagande politique dans le débat qui divise l'opinion canadienne au sujet de l'Union. La seconde porte plus directement sur la valeur littéraire. Huston admet implicitement que seule cette dernière devrait prévaloir, mais que le corpus canadien s'y prête mal. Il pardonne donc les faiblesses à une condition: «Mais au milieu des défauts de composition, et souvent des incorrections de style, le talent étincelle et brille comme l'électricité à travers de légers nuages ${ }^{2}$.» Le compilateur se fait fort de découvrir sous les maladresses de la jeunesse les véritables signes du talent.

Toutefois ces règles explicites ne justifient pas, à elles seules, les sélections des quatre volumes. En dressant un panthéon littéraire, Huston devenait le premier distributeur de la renommée. Non seulement il levait l'anonymat sur nombre de textes, mais il donnait aussi une idée de l'envergure de la production. Aussi imagine-t-on facilement les pressions de la part de quelques contemporains. Jusqu'à quel point demeure-t-il fidèle à ses principes? vii.

1. James Huston, Le répertoire national ou Recueil de littérature canadiennne (1848),

2. Ibid., v. 
Il est vrai que Huston élimine tous les textes qui pourraient faire croire à un parti pris dans le débat politique entre «unionnaires» et annexionnistes. Par exemple, il ne retient aucun des 17 poèmes que Louis-Thomas Groulx fait paraître dans L'Avenir parce qu'ils les jugent trop engagés. Des 27 poèmes déjà publiés par Joseph Lenoir en 1848 , il en choisit 6 dont les sujets sont plutôt anodins par comparaison avec d'autres, comme «Haine» ou «Aux sangsues du peuple», qui sont de violents réquisitoires contre les gens au pouvoir. On s'étonne toutefois qu'il ait retenu des poèmes, comme "Aux exilés politiques» de Joseph-Guillaume Barthe, qui ont déjà été censurés par les autorités civiles, ou encore les poèmes «Adieux à sir John Colborne» et «L'Union des Canadas» de P.-J.-O. Chauveau qui fustigent la politique de répression. Pourtant reconnues pour leur verve, aucune des chroniques de Napoléon Aubin dans Le Fantasque ne figurent au Répertoire. Huston était-il alors en brouille avec son ancien patron?

Le second critère de sélection devrait relever de la valeur littéraire des œuvres. Huston garde une certaine latitude pour excuser les faiblesses au profit des signes annonciateurs du talent. Ce critère, avouons-le, relève de la subjectivité. Pour respecter la hiérarchie des genres, il accorde beaucoup d'espace à la poésie. Si la valeur se mesure au nombre de lignes, c'est Joseph-Guillaume Barthe qui l'emporte avec 31 poèmes, suivi de F.-X. Garneau avec 19 et FrançoisMagloire Derome, avec 16. Personne ne songe à contester la place accordée à Garneau, mais il en va bien autrement de Barthe et de Derome. Pourquoi accueillir autant de poèmes médiocres du fougueux rédacteur de L'Aurore? Est-ce tout simplement parce qu'il en obtient plus facilement copie ou parce qu'il lui voue une admiration particulière? On serait plutôt tenté d'opter pour la première hypothèse d'autant que ses choix, dans le cas de Derome, tendent à la confirmer. En effet, plusieurs poèmes de ce dernier n'ont jamais paru ailleurs que dans Le Répertoire, comme le laissent croire les recherches de Jeanne d'Arc Lortie ${ }^{3}$.

Huston aurait donc reproduit les poèmes dont il disposait de préférence à ceux qu'il aurait pu trouver en dépouillant systématiquement les journaux. S'il avait vraiment eu du flair pour le talent, il aurait choisi plus que 6 des 23 poèmes que Joseph Lenoir avait déjà publiés, et il en aurait accepté plus que trois de Charles Lévesque qui en comptait déjà plus d'une vingtaine à son actif. Si l'espace consacré à chaque poète augure des réputations futures, il faut avouer que Huston

3. Jeanne d'Arc Lortie, Textes poétiques du Canada français; tome IV: (1838-1849) (Montréal, Fides, 1991), xl et xli. 
a misé sur les mauvais candidats. Barthe, Derome, Cherrier et les autres seraient tombés dans le plus complet oubli s'il ne les avait d'abord retenus.

Même s'il accorde la part du lion à la poésie, Huston garde un certain équilibre entre les différents genres littéraires. On peut même le soupçonner de chercher à contenter un peu tout le monde. C'est ainsi que des hommes qui ont très peu à voir avec la littérature ont droit à une mention: Georges Batchelor, copropriétaire de L'Avenir, George-Étienne Cartier, jeune étoile montante de la politique, et Thomas-J. Loranger, le futur juge. Deux extraits de sermons de Charles Chiniquy confirment la renommée du prédicateur de la tempérance. Avec six conférences, Étienne Parent occupe une part importante du quatrième volume.

Pour les nouvelles, Huston retient celles de Georges de Boucherville, de Philippe Aubert de Gaspé fils, de Charles-Wenceslas Dupont, de Joseph Doutre, d'Eugène L'Écuyer, de Charles Lévesque, de Louis-Auguste Olivier... Bref, son choix pour le genre narratif est cependant plus facile en raison de sa relative rareté. En effet, la production n'augmente que dans les années 1860. Par exemple, personne ne fera grief au compilateur d'ignorer les deux nouvelles d'Odile Cherrier parues dans Le Populaire en 1837 («Rosalie Berton» et «Adolphe et Eugène»). Entre «Faut-il le dire!» et «Le Frère et la Sœur» de Joseph Doutre, il opte pour le dernier avec raison. Il exerce le même discernement dans le cas de Georges Boucher de Boucherville: à «Louise Chawinikisique», il préfère «La Tour de Trafalgar», certainement plus littéraire. De tous les nouvellistes, c'est Eugène L'Écuyer qui lui offre le plus de choix, car il a déjà publié cinq nouvelles et un roman. Huston publie in extenso «La Fille du brigand», la meilleure œuvre de cet auteur, tout en accueillant deux autres nouvelles. Il inclut également La Terre paternelle de Patrice Lacombe, un autre roman ou longue nouvelle. On se demande pourquoi il a ignoré L'Influence d'un livre dont il se contente d'extraire les deux légendes, «L'Étranger» et «L'Homme de Labrador». Le roman de Philippe Aubert de Gaspé fils n'aurait cependant pas couvert plus d'espace que les deux précédents. Par ailleurs, on peut comprendre l'omission des Fiancés de 1812 et de Charles Guérin, trop volumineux.

Huston accorde également une place importante au théâtre malgré la rareté des pièces. Il en publie trois: Colas et Colinette de Quesnel, Le Jeune Latour d'Antoine Gérin-Lajoie et La Donation de Pierre Petitclair.

Malgré des choix critiquables, Le répertoire national contribue à façonner la première image de la littérature canadienne. En effet, 
jusqu'à l'avènement des collections de journaux en microfilms, il demeure longtemps tout ce que la critique peut consulter pour se former une idée de la littérature naissante. Réédité en 1893 par AdolpheBasile Routhier avec une nouvelle préface et des notes plus abondantes, il répond à un besoin des lecteurs qui s'interrogent sur les origines de la littérature canadienne. Une troisième édition verra le jour en 1976, mais sans aucun ajout. Peu après la publication du dernier volume, un éditeur parisien s'y intéresse particulièrement. Probablement peu charmé par les poésies, P. Jannet ne retient que les récits en prose qu'il publie en 1853 sous le titre Légendes canadiennes. Ainsi pour la première fois, quelques auteurs canadiensfrançais atteignent le public français.

Non seulement Le répertoire national a-t-il suscité une première collaboration entre un certain nombre d'écrivains, mais il a servi à définir le littéraire par rapport au non-littéraire. De ce fait, les auteurs qui y figurent acquièrent un début de réputation et, surtout, font l'objet du métadiscours littéraire qui sert de fondement à la littérature.

\section{LA RUCHE LITTÉRAIRE D'HENRI-ÉMILE CHEVALIER}

La Ruche littéraire répond mieux à la définition d'une revue. Elle comporte un certain nombre de chroniques et recense les productions récentes. Rédigée par le républicain français proscrit par le coup d'État du 2 décembre 1851, Henri-Émile Chevalier, qui a d'abord travaillé au Courrier des États-Unis, cette revue se propose de promouvoir la littérature canadienne en publiant autant que possible des œuvres d'auteurs locaux. Chevalier fait appel aux aspirants écrivains en leur promettant de recevoir leurs textes avec sympathie et compréhension. Mais il ne tarde pas à s'aliéner leur collaboration par l'arrogance et l'ironie mordante avec lesquelles il commente leurs écrits. Le rédacteur a beau s'excuser et promettre plus d'indulgence, il ne parvient pas à regagner la confiance de la gent lettrée et, après une première année, il se rend compte que la collaboration des Canadiens diminue considérablement.

Pourtant, il prêche d'exemple: dès son arrivée, il s'initie à l'histoire du Canada par la lecture de Garneau dont il fait une recension élogieuse. Il voit surtout dans cette histoire la geste des Français dans l'exploration de l'Amérique du Nord. S'il trouve là quelque sujet d'inspiration pour ses romans, il se sent d'abord attiré par les mœurs des indigènes que les succès de Fenimore Cooper ont déjà popularisés en Europe. Il y voit une belle occasion d'exploiter la vogue de l'exotisme indigène toujours vigoureuse en France. Il se fait la main par la traduction des Trappeurs de la Baie d'Hudson de Robinson et par 
l'édition du Grand Voyage du pays des Hurons de Sagard ${ }^{4}$. Voilà un filon qu'il voudrait voir exploiter par les Canadiens.

En 1853, il a pu bénéficier du milieu de l'Institut canadien alors en pleine effervescence pour recruter des collaborateurs. C'est là qu'il rencontre Joseph Lenoir, Georges Batchelor, Charles Laberge, Georges de Boucherville qui lui fournissent des poèmes et des nouvelles. D'autres littérateurs répondent aussi à son appel. Ce sont des jeunes gens sans appartenance particulière, comme Orphir Peltier, organiste à l'église Saint-Patrick de Montréal, Félix-Gabriel Marchand, en stage de clerc à Saint-Jean-sur-le-Richelieu, James MacPherson Le Moyne, L.-J.-C. Fiset, Eugène L'Écuyer et Malvina $\mathrm{D}$. de la région de Québec.

On peut croire que, par sa propre production, Chevalier tente d'orienter sa revue. Un premier récit tiré de Garneau, l'île de sable, inaugure son œuvre romanesque. En 1854, il fait paraître, en feuilleton dans La Patrie, La Batelière du Saint-Laurent. Un épisode de la guerre de 1812. L'année suivante, il livre au Moniteur canadien son Jacques Cartier ou le Premier Jour de l'an 1536 au Canada. Chevalier apparaît ainsi comme le véritable initiateur du roman historique au Canada. Bien avant les Soirées canadiennes, il ouvre à l'imaginaire local une forme d'affabulation fondée sur le passé national, sans toutefois parvenir à susciter des émules.

Il désapprouve l'orientation de P.-J.-O. Chauveau. Charles Guérin, roman que Cherrier vient de publier en volume, reste pour lui une énigme: «Enfin, nous devons l'avouer, nous avons vainement tenté de découvrir le but de l'ouvrage; il n'existe pas ${ }^{6} . »$ Eugène L'Écuyer, dont il admire le talent, se limite aux aspects moraux des comportements humains. La production locale lui paraît donc décevante et peu propice à son dessein.

Mais le rédacteur ne s'en attriste pas outre mesure, car il recherche plutôt, quoi qu'il en dise, la promotion de la littérature française dans la diaspora américaine. Il rêve de regrouper, dans une sorte de société des gens de lettres, tous les écrivains francophones du Nouveau Monde, peu importe leur domicile. Il déplore l'isolement de chacun: «Le malheur est que les écrivains français en Amérique, ne se connaissent presque point, et qu'au lieu de former un faisceau, ils luttent isolément jusqu'à ce que le dégoût ou le désespoir les ait jetés hors de

4. Gabriel Théodat Sagard, Le Grand voyage du pays des Hurons [...], édité par HenriÉmile Chevalier (Paris, Librairie Tross, 1853), 2 vol.

5. Le Moniteur canadien, 8,15 (4 janvier 1855): 1.

6. La Ruche littéraire, $1^{\text {ere }}$ série (1853), 106. 
la voie littéraire ${ }^{7} . »$ Il projette de faire de sa revue le trait d'union entre eux pour susciter un renouveau de la littérature française en Amérique.

Avec le temps, Chevalier a de plus en plus de mal à remplir les 64 pages qu'il a promises à ses lecteurs pour chaque numéro. Les livraisons s'espacent, malgré les efforts qu'il déploie pour en rédiger une bonne partie. Mais au lieu de reproduire simplement des articles de revues françaises, selon une pratique courante dans les journaux d'alors, il se tourne vers un réseau d'écrivains français en terre d'Amérique. Il requiert la collaboration de l'agronome Frédéric Ossaye, auteur des Veillées canadiennes, du vétérinaire Félix Vogeli, son compagnon d'exil, de Jean-Sylvain Gentil, un autre proscrit réfugié à la Nouvelle-Orléans, de Victor Baron et Van Hoven, tous les deux journalistes français à New York.

Malheureusement, un incident de parcours l'empêche de poursuivre son rêve. En 1855, son imprimeur quitte précipitamment le pays avec l'argent des abonnements. C'est un dur coup pour Chevalier qui vient de racheter sa part. Le proscrit met près de quatre ans pour se refaire financièrement et reprendre en 1859 la publication de sa revue. Échangée avec New York, Paris et la Nouvelle-Orléans, $L a$ Ruche littéraire revêt un caractère plus international que canadien. Chevalier se vante de publier la seule revue littéraire française en Amérique.

On peut croire que, faute d'avoir su attirer la collaboration des Canadiens, Chevalier, à la reprise de sa revue, ne cache plus son orientation en faisant de son périodique «un écho de la France» pour contribuer au progrès de la littérature française dans le Nouveau Monde. Mais, son manque de moyens, pécuniaires surtout, l'oblige, en juin 1859, à suspendre la parution de sa revue. Il profite de l'amnistie que Napoléon III accorde aux proscrits pour rentrer en France.

L'échec de Chevalier s'explique de diverses façons. Le manque d'argent y est certes pour beaucoup, mais son incompréhension des Canadiens compte davantage. À l'époque où ces derniers tentent de définir leur identité nationale par une littérature qui leur soit propre, Chevalier leur propose de se considérer ni plus ni moins comme n'importe quel autre émigré français en Amérique. En faisant abstraction de deux siècles de vie autonome, il s'aliène les Canadiens qui ne peuvent plus revenir dans le giron français, comme si rien ne s'était passé.

7. La Ruche littéraire, $3^{\mathrm{e}}$ série (1859), 47. 


\section{LE JOURNAL DE L'INSTRUCTION PUBLIQUE}

Le 15 janvier 1857, une circulaire annonce la publication de deux périodiques pédagogiques, l'un de langue française et l'autre de langue anglaise, relevant tous deux du surintendant de l'Instruction publique. Ils jouissent d'une subvention annuelle de $450 £$, ce qui en fait les premières revues subventionnées. Il s'agit en principe de revues pédagogiques, mais le surintendant a des visées plus larges. Sous prétexte que le milieu restreint de l'éducation ne permet pas la spécialisation, comme c'est le cas dans les pays plus populeux, il se propose de «combiner dans des proportions voulues, tous les éléments qui doivent composer un journal de l'Instruction publique, répandre dans tout le pays sous une forme agréable les connaissances utiles, inspirer à la jeunesse le goût des saines lectures, complément des bibliothèques paroissiales ${ }^{8}$ ».

Homme de lettres avant tout, P.-J.-O. Chauveau conserve la main haute sur le périodique et prend des libertés avec une formule pédagogique trop rigide. Certes, la revue fournit aux instituteurs des exercices d'analyse grammaticales, des sujets de composition, des corrections de locutions vicieuses, mais elle livre aussi des nouvelles du monde littéraire et scientifique, des bibliographies et des biographies. En fait, Chauveau désire établir un premier lien entre l'école et la littérature naissante. Il invite les jeunes écrivains à lui faire parvenir des textes, et souvent les poèmes à apprendre par cœur sont de Joseph Lenoir, de F.-X. Garneau, de Louis-C. Fiset, d'Adolphe Marsais, de Louis Fréchette et de Benjamin Sulte. Par la même occasion, il entend répandre le goût de l'histoire du Canada: «À ce propos, nous pouvons annoncer que des documents précieux sur l'archéologie, l'histoire et la biographie canadiennes ont été mis à notre disposition et que nous en ferons part de temps à autre à nos lecteurs, sous le titre de 'Fragments historiques'. .»

La chronique bibliographique comporte des recensions de livres et un bulletin des publications et des réimpressions les plus récentes. Elle rend compte parfois de plusieurs livres par mois et fait pour cela certainement appel à une équipe de recenseurs qui gravitent autour de la librairie Crémazie. Malgré leur superficialité et leur caractère adulateur, ces recensions constituent une amorce de critique littéraire. Elles se distinguent de celles des journaux par une analyse de contenu qui atteste d'une lecture attentive. C'est surtout par la «Petite Revue mensuelle» que Chauveau rend compte de la vie littéraire. Il signale les

8. Le Journal de l'Instruction publique, éditorial, janvier 1857.

9. P.-J.-O. Chauveau, «Au public canadien», Le Journal de l'Instruction publique, 1,1 (1857): 11. 
réunions savantes, les conférences, les visites d'écrivains étrangers... Il parvient ainsi à rendre compte d'une manière intéressante de la vie littéraire.

Certes, les avis officiels, les documents de toutes sortes sur l'instruction publique et les nombreux tableaux statistiques tendent à émousser ce caractère littéraire, mais ses destinataires premiers ne le perçoivent pas ainsi. En effet, les instituteurs, qui se sentent laissés pour compte par une revue aux objectifs trop élevés, fondent leur propre revue en 1864 pour défendre leurs propres intérêts. La Semaine consacre la désaffection des enseignants à l'égard d'une revue plus littéraire que pédagogique. Il devenait évident que Chauveau se servait des fonds publics pour soutenir une revue que la gent littéraire ne pouvait pas se payer.

\section{L'ÉCHO DU CABINET DE LECTURE PAROISSIAL}

L'Écho du Cabinet de lecture paroissial souffre de la même ambiguïté que le Journal de l'Instruction publique. À mesure que monte la tension entre l'Institut canadien et Montréal et $\mathbf{M}^{\mathrm{gr}}$ Ignace Bourget, le parti clérical sent l'urgence de lancer une contre-attaque. Grâce aux moyens dont ils disposent, les sulpiciens ouvrent, le 2 février 1857, le Cabinet de lecture paroissial pour accueillir et encadrer les dissidents de l'Institut canadien. Bientôt doté d'un édifice magnifique comprenant une bibliothèque, une salle des périodiques et une salle de conférence, le Cabinet s'identifie comme un regroupement d'ultramontains. Les clercs français récemment arrivés de France y prennent un ascendant incontestable: Louis Regourd, Adam-Charles Desmazures, Dominique Granet, Antoine Giband, André Nercam, Hyacinthe Rouxel et Louis Colin sont autant de noms qui s'imposent par la fermeté de leur plume, la rigueur de leur argumentation et le prestige de leur autorité. Ils ont l'avantage sur les prêtres canadiens d'une formation cléricale complète d'après la formule sulpicienne - les Canadiens pour la plupart ont appris leur théologie par eux-mêmes pendant leurs années de régence. Au courant du grand brassage d'idées qui secoue la France, ces prêtres adoptent une attitude d'extrême droite qu'ils tâchent d'inculquer à leurs jeunes recrues.

Pour agrandir le rayonnement du cercle, le directeur Regourd décide, en 1859 , de publier un bulletin particulièrement destiné à diffuser les conférences qui s'y donnent. Craignant de rebuter le grand public par des sujets trop arides, il y insère «quelques pièces étrangères prises çà et là dans les écrivains connus et dont le mérite peut ajouter quelque prix à ce recueil et plaire par leur variété ${ }^{10} \gg$. La nou-

10. Louis Regourd, «Avis très important», $L^{\prime}$ Écho du Cabinet de lecture paroissial, 1,1 (15 janvier 1859): 1 . 
velle feuille entend donc occuper un créneau intermédiaire entre la revue et le recueil pour atteindre un plus grand public. De plus, elle affiche sa préférence pour des textes français, laissant dans l'ombre la promotion de la littérature nationale.

Cette feuille jouit quand même d'un excellent réseau de distribution: «L'Écho trouvera sa place dans toutes les maisons d'éducation, collèges, pensionnats, bibliothèques paroissiales et autres, enfin dans toutes les familles jalouses d'inspirer à leurs enfants l'amour de la Religion et le goût de la saine lecture ${ }^{11}$.» Elle sait mettre à profit le réseau institué par le clergé pour mieux contrôler les lectures.

Les conférences de fond, vraiment porteuses de messages, sont signées par des sulpiciens et des jésuites. Elles traitent des questions mises de l'avant par les libéraux: la liberté, la tolérance, l'opinion publique, les lectures... Les débats, comme «plaidoyer pour la proéminence des quatre arts libéraux, éloquence, poésie, musique, peinture», semblent un os jeté en pâture aux jeunes fauves avides de se faire les dents. Ces derniers semblent si bien endoctrinés qu'ils calquent leur argumentation sur celle de leurs maîtres.

Pour agrémenter ces plats de résistance parfois indigestes, la direction sert des aliments plus légers: quelques poésies, des récits de voyage, des fables et même quelques feuilletons, mais de provenance française. Le choix des articles se fait en fonction de trois groupes de lecteurs: les conférences pour les hommes formés et les étudiants, les poésies et les fables pour les enfants et les feuilletons pour les femmes. L'intérêt de ces dernières importe au plus haut point puisque ce sont elles qui dirigent les lectures au foyer. Aussi des feuilletons, comme «Lettre à ma fille» de la comtesse de Bassanville et «la Maman de huit ans» de la comtesse de Colmar leur sont-ils particulièrement destinés.

Malgré une teneur nettement littéraire, L'Écho du Cabinet de lecture paroissial favorise peu la diffusion des écrits des écrivains canadiens. Une conception trop immédiatement ordonnée au prosélytisme réduit la littérature à une sorte d'apostolat, comme le signale à juste titre Paul Stevens dans sa conférence du 15 mars 1859: «Tous les charmes de l'art d'écrire, toutes les ressources d'une féconde imagination, tous les ornements ingénieux du langage, qui ne voilent nos pensées que pour les faire paraître plus belles, doivent être employés à rendre les hommes meilleurs ${ }^{12}$.» Ainsi, loin de chercher à affirmer leur originalité, les divers Canadiens qui y prennent la plume tentent

11. Louis Regourd, «À nos lecteurs», ibid., 1,3 (1er février 1859): 33.

12. Paul Stevens, «Esquisses des mœurs», ibid., 1,10 (15 mai 1859): 147. 
d'apporter leur concours à la lutte contre le libéralisme et témoignent de leur soumission à l'autorité ecclésiastique.

\section{LES SOIRÉES CANADIENNES ET LE FOYER CANADIEN}

La fondation de la revue Les Soirées canadiennes, en 1861, marque aux yeux de plusieurs le début d'un mouvement littéraire qui aurait donné à la littérature canadienne sa véritable impulsion. Mais est-ce là seulement un effet de discours ou y trouve-t-on un fondement dans la réalité?

L'abbé Henri Raymond Casgrain pourrait être à la naissance de ce mythe, car c'est lui surtout, avec son étude sur «le Mouvement littéraire de $1860^{13}$ », qui a formulé le discours le plus pertinent sur le sujet. Toutefois, sa bonne foi peut être sujette à caution en raison de sa propension à exagérer son rôle. Aussi, n'est-il pas inutile de réexaminer comment se présentait la vie littéraire à Québec avant son arrivée.

Dès les années 1840, la jeunesse lettrée de Québec se réunissait déjà de façon impromptue, soit à l'Hôtel de la Tempérance, soit à l'Hôtel de la Cité, soit dans les locaux du Fantasque. S'y retrouvaient P.-J.-O. Chauveau, Joseph-Charles Taché, Télesphore Fournier, James Huston, Auguste Soulard, Marc-Aurèle Plamondon, Napoléon Aubin et quelques autres. Ces réunions spontanées mènent à la fondation, le 4 octobre 1843, de la Société canadienne d'études littéraires et scientifiques qui regroupe, au cours de son éphémère existence, une trentaine de membres. Le retour de Louis-Joseph Papineau en politique sème cependant la division parmi les sociétaires. Certains se rangent du côté des «Rouges» et fondent Le National (1855), un journal qu'animent Télesphore Fournier, Gabriel Huot et Marc-Aurèle Plamondon et qui livrera une lutte sans merci au Canadien et au Journal de Québec. Pendant les années 1850, un autre groupe se forme au hasard des rencontres. Des «anciens» fréquentent le magasin de Charles Hamel, rue Saint-Jean. Ce sont des vétérans des affaires, comme John Fraser, Henry Forsyth et Benjamin Lemoine; des amateurs d'histoire, comme F.-X. Garneau, G.-B. Faribault et P. Aubert de Gaspé pour ne mentionner que les plus connus. «La conversation, comme en témoigne Hubert Larue, ne languissait guère: mais certains sujets repris, puis mis de côté, avaient le privilège de faire battre les cœurs plus vite, tels: la guerre de 1812 - de Salaberry - le général Brock - puis des conversations intarissables sur les antiquités de

13. Abbé Henri-Raymond Casgrain, «Le mouvement littéraire de 1860», Le Foyer canadien, 6 (1866): 1-32. 
Québec et de ses environs ${ }^{14} . »$ Des bibliophiles se rencontrent à la librairie des frères Crémazie et prolongent leur conversation dans l'arrière-boutique. S'y retrouvent à l'occasion Philippe Aubert de Gaspé, François-Xavier Garneau, Jean-Baptiste Ferland, JosephCharles Taché, Antoine Gérin-Lajoie et leur hôte, Octave Crémazie. Aucune réunion régulière n'est prévue, et personne ne songe à prendre la direction du groupe. Pourtant, c'est de ce dernier groupe que naîtront Les Soirées canadiennes, une revue qui révèle un certain niveau de concertation.

Les historiens ont spontanément désigné l'abbé Casgrain comme chef et mentor du groupe en se fiant à sa version des faits. Mais jusqu'à quel point peut-on croire celui qui a eu l'avantage sur les autres membres de récupérer le discours à son profit? En fait quel écrivain, à part Casgrain, jouissait alors du prestige nécessaire pour assumer un pareil leadership?

Parmi les familiers de la librairie Crémazie, F.-X. Garneau l'emportait certainement en renommée sur tous les autres. Déjà connu comme poète, il avait acquis une réputation internationale avec la publication de son Histoire du Canada. La troisième édition passablement remaniée lui ralliait maintenant la majorité des appuis. Mais l'historien n'était pas un homme d'équipe: travailleur solitaire, il se laissait difficilement embrigader. Le prestige de Ferland égalait alors aux yeux de ses contemporains, celui de Garneau. Dans sa correspondance, Crémazie les place sur un même pied: «Nous n'avons donc réellement que deux œuvres hors ligne, les monuments élevés par MM. Garneau et Ferland ${ }^{15}$.» Titulaire de la chaire d'histoire à l'Université Laval, Ferland s'est surtout fait connaître par ses cours publics d'histoire du Canada qui ont attiré un auditoire nombreux. Leur publication en 1861 couronne sa carrière. Aux yeux d'Antoine GérinLajoie, son ancien élève, il apparaît comme un maître à penser capable d'orienter un groupe. Plus homme de cabinet qu'homme d'action, il oriente plus qu'il ne dirige.

Ancien député, commissaire du Canada à l'exposition universelle de Paris, en 1855, Joseph-Charles Taché s'est fait connaître par des ouvrages comme Le Canada et l'exposition universelle de 1855, De la tenure seigneuriale en Canada, Des provinces de l'Amérique du Nord et d'une union fédérale. Sa Pléiade rouge lui a valu, en 1858, une réputation de pamphlétaire. Avec Hector Langevin, il a lancé en 1857 un nouveau journal, Le Courrier du Canada, qui rallie autant les ultra-

14. Hubert Larue, Voyage sentimental sur la rue Saint-Jean (Darveau, 1879), 99.

15. Octave Crémazie à H. R. Casgrain, H. R. Casgrain, Euvres complètes (Montréal, Beauchemin et Valois, 1865), II: 348. 
montains que les conservateurs. C'est un homme d'action capable de susciter le militantisme. Il joue, à n'en pas douter, un rôle déterminant dans la fondation de la nouvelle revue. L'hôte du groupe, Octave Crémazie, n'est certes pas à négliger. Fin causeur doté d'une érudition remarquable, il adore les livres et en parle d'abondance. Les lettrés trouvent en lui un interlocuteur de choix. Mais il y a plus. Depuis la publication de ses poèmes dans L'Ami de la religion, Crémazie s'est acquis une réputation de poète national. C'est lui qui a montré le meilleur parti à tirer pour la poésie de l'Histoire de Garneau. Avant même qu'on ait défini ce que devait être la littérature nationale, Crémazie offrait en ses vers le modèle à suivre. Malgré une certaine propension à la forfanterie, il n'est toutefois pas de nature à prendre des initiatives.

Philippe Aubert de Gaspé, encore sans publication, n'a cependant pas attendu la parution du premier numéro des Soirées canadiennes pour se mettre à écrire ${ }^{16}$. Il entretient volontiers ses interlocuteurs de son projet de roman, comme le prouvent les extraits publiés dans Les Soirées canadiennes de 1862 , probablement à la demande de J.-C. Taché. P.-J.-O. Chauveau, redevenu simple député depuis 1855 , fréquente assidûment la librairie Crémazie et compte sur son prestige littéraire pour relancer sa carrière. L'édition de son roman par G.-H. Cherrier, en 1853, l'accrédite comme conférencier et comme orateur dans plusieurs institutions. Mais bientôt sa nomination comme surintendant de l'Instruction publique l'amène à quitter Québec pour Montréal.

Henri-Raymond Casgrain serait celui qui, selon la tradition, aurait pris la direction du groupe et l'aurait amené à fonder Les Soirées canadiennes $^{17}$. En 1859, à l'époque où il arrive à Québec comme vicaire de la paroisse Notre-Dame, il n'a que 28 ans et n'a signé aucune œuvre. Il en est encore à la rédaction de ses Légendes canadiennes. C'est avec déférence qu'il reconnait l'accueil cordial que lui réservent ses aînés:

Dans notre jeune pays, où la littérature est encore dans l'enfance, il ne peut être question de gloire littéraire. On peut prétendre tout au plus à la notoriété. Je commençais à y arriver et cet avantage me valait d'être accueilli avec bienveillance par les hommes qui s'étaient déjà fait un nom dans le pays. Je ne puis penser sans un 254.

16. Luc Lacourcière, «L'enjeu des Anciens Canadiens», Cahier des dix, 32 (1967): 223-

17. Réjean Robidoux, «Les Soirées canadiennes et Le Foyer canadien dans le mouvement littéraire québécois de 1860", Revue de l'Université d'Ottawa, 28,4 (octobre-décembre 1958): 411-452. 
profond sentiment de gratitude aux encouragements et aux conseils que me prodiguait leur amitié et dont je comprends mieux la sagesse maintenant que j'en ai l'expérience ${ }^{18}$.

Aussi n'est il pas surprenant que Casgrain offre en hommage à «J.-C. Taché, chevalier de la Légion d'honneur», sa troisième légende publiée dans le Courrier du Canada ${ }^{19}$, reconnaissant ainsi l'hégémonie de Taché sur le groupe. De tous les membres du groupe, Casgrain est certainement celui qui se rend le plus compte de l'existence d'un champ littéraire et de l'importance de l'investir. Homme de beaucoup d'entregent, il excelle dans les relations humaines, ce qui n'en fait pas pour autant un homme d'action. Il le montrera bien au cours des six années d'existence des deux revues. Il abandonne à Antoine Gérin-Lajoiec comme à son second, le soin de prendre des initiatives et de régler les questions pratiques.

En 1859, Gérin-Lajoie revient à Québec en compagnie de son beau-père, Étienne Parent, après une absence de quatre ans. Québécois d'adoption seulement, il ne jouit pas encore d'intelligences dans la place. Toutefois ses juvenalia lui valent une certaine renommée, car c'est surtout comme rédacteur de La Minerve et comme membre de l'Institut canadien qu'il s'est fait connaître. Sa récente nomination comme bibliothécaire adjoint du Parlement lui assure un prestige indiscutable dans le cercle des intellectuels. Il compte aussi sur l'appui et l'estime de son ancien maître, J.-B.-A. Ferland, et de son beau-père, Étienne Parent. Mais son capital symbolique encore assez mince ne l'autorise pas à prendre la direction du groupe de la librairie Crémazie et à imposer ses vues. Il projette plutôt de fonder une revue littéraire avec son frère Elzéar et son cousin Évariste Gélinas. Le projet aurait quand même une allure nationale dont il fait part à son ami Bellemare:

[...] si pourtant tous ceux qui peuvent écrire dans le pays s'unissaient pour faire un bon recueil périodique, écrit dans un bon esprit, il me semble que la jeunesse canadienne en retirerait quelque avantage. La grande affaire serait d'obtenir l'encouragement de la classe intelligente et éclairée de Montréal et de Québec. Il faudrait que ce fût une œuvre nationale sans quoi elle n'aurait aucune chance de réussite ${ }^{20}$.

Dans ce dessein, le bibliothécaire entre en contact avec E.-R. Fréchette qui lui propose d'acheter le journal Le Canadien et son

18. H. R. Casgrain, Souvenances canadiennes, III: 39.

19. Le Courrier du Canada, 4,90 (30 septembre 1860).

20. A. G. L. à R. Bellamare, Québec, 9 novembre 1859, enveloppe 6, pièce 5356-2, cité par R. Dionne, Antoine Gérin-Lajoie, homme de lettres (Sherbrooke, Éditions Naaman, 1978), 236. 
imprimerie pour la somme de 2000 louis. L'opération se financerait d'elle-même par la revente de l'imprimerie. Il ne manque plus qu'une équipe de collaborateurs. L'initiateur du projet sollicite son ami Bellemare de lui soumettre quatre ou cinq noms de ses amis. Il mise aussi sur la collaboration de Louis-Wilfrid Marchand, leur ami commun, et sur celle d'Étienne Parent. Mais ce dernier lui fait valoir l'impossibilité pour des fonctionnaires de prendre une part active à la rédaction d'un journal politique ${ }^{21}$. Gérin-Lajoie, toujours très intime avec l'abbé Ferland, lui fait certainement part de son intention. De leur côté, les habitués de la librairie Crémazie mijotent-ils un projet similaire? Rien ne saurait le confirmer. L'hypothèse la plus vraisemblable voudrait que le groupe de Québec ait spontanément adopté le projet de Gérin-Lajoie. Taché, déjà familier avec le monde des imprimeurs de Québec, se serait entremis pour les arrangements avec les frères Brousseau. On peut donc vraisemblablement croire que l'initiative de fonder une revue revient à Gérin-Lajoie.

C'est au moment de déterminer l'orientation de la revue que se manifestent les premières luttes pour la domination du champ. Tout en prônant un recueil de littérature nationale, Gérin-Lajoie et Taché s'opposent à propos de l'extension à donner au terme littérature. Tout au long de sa carrière, l'auteur de Jean Rivard insiste sur le caractère utile des lettres par opposition au caractère frivole de la fiction. Plusieurs années auparavant, dans un débat à l'Institut canadien sur le sujet suivant: «Les Lettres ont-elles été plus nuisibles qu'utiles à la société?», il a signifié qu'il entendait la littérature au sens très large du XVIII ${ }^{e}$ siècle, à savoir qu'elle comprenait autant les sciences que les lettres. Aussi préférait-il orienter la revue vers des genres sérieux comme l'histoire, l'économie, le droit et les sciences de la nature. Taché, pour sa part, se réfère à une définition beaucoup plus romantique de la littérature; il croit que le génie particulier des peuples se manifeste principalement dans des genres populaires comme les contes, les légendes et les chansons:

Ces légendes et ces contes, dans lesquels les peuples ont versé leur âme, avec lesquels ils ont cherché à satisfaire, dans certaines limites, ce besoin du merveilleux qui est le fond de notre nature; ces souvenirs réels ou fictifs, attachés à tel ou tel endroit de chaque pays habité, constituent une portion notable, le fonds on peut dire de toute littérature nationale ${ }^{22}$.

21. Antoine Gérin Lajoie à Raphaël Bellemare, lettre du 5 septembre 1860, enveloppe 6, pièce 5356-II, cité par R. Dionne, Antoine Gérin Lajoie, homme de lettres, 237.

22. Joseph-Charles Taché, Forestiers et Voyageurs (Montréal, Fides, 1981), 15. 
C'est d'abord par son imaginaire qu'un peuple exprime son génie propre, imaginaire certes des grands artistes et des grands écrivains, mais aussi imaginaire collectif, peut-être moins délicat, mais plus authentique et sûrement plus prometteur d'originalité. À ce titre, les Canadiens, bien qu'encore sans littérature et sans beaux-arts, jouissent de l'avantage de pouvoir compter sur une tradition orale extrêmement riche. Aussi est-ce un genre d'opération anthropologique que propose Taché en adaptant une phrase de Nodier: «Hâtons-nous de raconter les délicieuses histoires du peuple avant qu'il ne les ait oubliées ${ }^{23}$.»

Bénéficiant d'une position hégémonique dans le groupe, Taché l'emporte sans doute avec l'appui de Casgrain et de Hubert Larue. L'auteur de «La Jongleuse», qui a déjà flairé la bonne affaire, est alors en pleine rédaction de ses Légendes canadiennes. Quant à l'auteur des Anciens Canadiens, il manifeste son intérêt pour le folklore. Larue opine dans le même sens, comme le montreront son Voyage autour de l'île d'Orléans et ses «Chansons historiques du Canada». Gérin-Lajoie se rend aux désirs de ses collègues sans renoncer pour autant à sa notion de littérature utile. Il entreprend la rédaction de son Jean Rivard, le défricheur pour illustrer ce qu'il entend par là. Ces divergences de vues n'expliquent pas, à elles, seules la scission qui survient au sein du groupe quelques années plus tard, mais elles n'y sont pas étrangères. Dans une seconde manche, Gérin-Lajoie et l'abbé Ferland l'emporteront en vouant Le Foyer canadien aux études historiques surtout.

Le mot d'ordre donné par Taché suffit-il pour que l'on parle d'une école, comme plusieurs historiens de la littérature le prétendent? Les écoles littéraires, depuis la Pléiade, se sont généralement signalées par un manifeste qui exprimait leur volonté de rupture. Par la suite, leurs membres se sont appliqués dans leurs œuvres à en illustrer les principes. Il va sans dire que les Préfaces de Cromwell et de Mademoiselle de Maupin admettaient une certaine dissidence puisque les grands écrivains obéissent plus à leur génie qu'aux règles.

Le groupe de la rue de la Fabrique n'a jamais publié de manifeste autre que celui de l'abbé Casgrain intitulé «Le Mouvement littéraire de 1860», rédigé en 1866, à la veille même de sa dispersion. Dans une rétrospective, le critique fait le bilan des cinq premières années et ouvre des perspectives sur l'avenir de la nouvelle littérature. Il tente surtout, par des comparaisons avec la littérature française, de fixer un ordre de grandeur au phénomène littéraire d'ici. L'abbé Casgrain s'y donne évidemment un rôle de premier plan. C'est avec humour 
qu'Adolphe-Basile Routhier pose la question: «M. l'abbé Casgrain déclare que l'avenir de la littérature canadienne est assuré depuis 1860. Je me suis demandé pourquoi cette date plutôt qu'une autre, et je me suis aperçu que cette année-là (1860) avait vu paraître [s]es Légendes canadiennes ${ }^{24}$.»

Le prospectus des Soirées canadiennes, par sa brièveté, n'a guère l'allure d'un manifeste, mais il n'en définit pas moins une orientation précise: «Ce recueil sera surtout consacré à soustraire nos belles légendes canadiennes à un oubli dont elles sont plus que jamais menacées, à perpétuer ainsi les souvenirs conservés dans la mémoire de nos vieux narrateurs, et à vulgariser la connaissance de certains épisodes peu connus de l'histoire de notre pays $^{25} . »$ Ces quelques lignes énoncent tout un programme. Elles mettent d'abord l'accent sur la récupération: «soustraire à l'oubli». Aux yeux des signataires, le pays traverse une phase climatérique. L'école à la portée de tous et les perspectives d'alphabétisation générale menacent de disparition la tradition orale. Bientôt le peuple aura perdu l'essentiel de son patrimoine culturel. L'écrit suppléera à la mémoire collective, ou du moins en prendra le relais.

Est-ce à dire que les écrivains se verront réduits à un simple rôle de transcripteurs? On serait porté à le croire si on se limite au texte du prospectus. Mais en réalité, ils l'entendent autrement. Taché, Casgrain et Gaspé, non contents de colliger, réinterprètent le folklore à la lumière de la tradition littéraire. En d'autres mots, ils choisissent l'imaginaire populaire comme source privilégiée d'inspiration littéraire. Tout en prétendant le préserver, ils le modifient en fonction de leur idéologie de classe. Taché admet implicitement que la simple transcription serait insatisfaisante: «Je voudrais pouvoir leur laisser [aux contes populaires] ce ton de franche gaieté, de naïveté charmante, de philosophie primitive et d'allégorisme souvent profond que prennent, tour à tour, les récits populaires ${ }^{26} »$, mais un tel idéal ne s'atteint que par l'art. Aussi admet-il quelques lignes plus bas: «Dans l'Histoire du père Michel, j'ai réuni sur la tête d'un seul acteur plusieurs aventures qui sont réellement advenues à divers personnages que j'ai connus ${ }^{27}$.» C'est ainsi que le père Michel s'introduit dans le monde littéraire, non pas en tant que témoin d'un patrimoine culturel,

24. Auguste Laperrière, comp., Les Guêpes canadiennes (Ottawa, A. Bureau, iprimerie, 1881), 263.

25. «Prospectus des éditeurs», Soirées canadiennes, (Québec, Brousseau \& Frères éditeurs, 1861), I: i-iii.

26. Jean-Charles Taché, Forestiers et Voyageurs (Montréal, Fides, 1981), 16.

27. Loc. cit. 
mais en tant que narrateur délégué de l'auteur. Jusqu'à quel point la tradition orale ainsi transformée présente-t-elle au peuple sa propre image? L'entreprise de pédagogisation du folklore ne risque-t-elle pas d'en changer foncièrement la nature?

L'autre volet du programme des Soirées canadiennes porte sur la vulgarisation de l'histoire: "vulgariser la connaissance de certains épisodes peu connus de l'histoire de notre pays $^{28} . »$ De nouveau, l'aspect pédagogique prédomine. Évidemment, la grande histoire relève des historiens comme Garneau et Ferland et elle demande à être lue dans leur texte. Mais il en va autrement de la petite histoire, de celle qu'affectionnent les amateurs de vieilles choses dans leurs réunions au magasin Hamel. Elle pique la curiosité et divertit tout en instruisant. C'est ainsi qu'on espère amener les lecteurs moins sérieux à s'intéresser à la grande histoire. Le processus pédagogique mis de l'avant justifie le roman historique, l'autre volet du programme.

Énoncé de façon laconique dans le prospectus des Soirées canadiennes, ce programme a été bel et bien compris et interprété en ce sens par les collaborateurs de la revue. Les œuvres qui en résultent le témoignent. Taché est peut-être celui qui a visé le plus directement cet objectif avec ses Trois légendes de mon pays et Forestiers et Voyageurs. L'abbé Casgrain travaillait dans le même sens, comme en témoignent ses Légendes canadiennes. Dans son Voyage autour de l'île d'Orléans, Hubert Larue recense le folklore qui s'attache à ces lieux. Il dévoile un autre pan du patrimoine national dans ses «Chansons populaires et historiques du Canada». Aubert de Gaspé marque son accord avec le groupe en fournissant aux Soirées canadiennes les extraits les plus significatifs en ce sens des Anciens Canadiens. Ernest Gagnon valorise également le folklore dans Chansons populaires $d u$ Canada. Cette floraison ne serait donc pas le fruit du hasard ou d'une simple mode, mais le produit de la concertation.

De tous les romans de l'époque, Les Anciens Canadiens répond le mieux à l'esprit de l'école. Bien que résidant à Montréal, Georges Boucher de Boucherville se laisse influencer par le groupe de Québec et refond son roman Une de perdue, deux de trouvées pour lui donner dans sa seconde partie une allure historique. Napoléon Bourassa obéit au même impératif dans Jacques et Marie, une histoire romancée de la déportation des Acadiens. Ce sera surtout Joseph Marmette qui exprimera le mieux l'esprit du groupe: dès 1866 il livre à La Revue canadienne de Montréal son roman Charles et Éva qui s'applique à romancer un épisode de l'Histoire de Garneau.

28. «Prospectus des éditeurs», Soirées canadiennes, I: i. 
Cette orientation, principalement donnée par Taché, ne recueille cependant pas tous les suffrages. Antoine Gérin-Lajoie profite de la scission au sein du groupe pour revenir à son idée de départ. Dans le prospectus du Foyer canadien, il écrit:

Ce recueil, destiné à réunir et à conserver nos essais de littérature indigène, sera consacré à la publication d'œuvres inédites: poésies, critiques littéraires, légendes, nouvelles, pourvu qu'elles soient de fidèles peintures de mœurs et de la nature de notre pays, impressions de voyage, esquisses historiques, bibliographiques et même topographiques, et enfin toute œuvre canadienne se distinguant par quelque originalité de vues, de pensée ou de style ${ }^{29}$.

Déjà on perçoit qu'un sérieux embargo pèse sur les œuvres d'imagination: l'obligation faite aux auteurs de respecter une réalité canadienne de convention. Ce n'est cependant que plus loin dans le prospectus que la direction dévoile ses véritables priorités: «[...] n'avons-nous point, dans nos archives et nos bibliothèques publiques ou privées, exposés à des accidents de toutes sortes, des documents manuscrits de la plus grande valeur et du plus haut intérêt? N'avonsnous pas d'anciens ouvrages dont la réimpression serait désirable ${ }^{30}$ ?» $\mathrm{Ne}$ voit-on pas prévaloir la préférence de l'abbé Ferland pour les documents historiques? C'est dans cet esprit que la revue publie «Notice biographique sur Monseigneur Joseph Octave Plessis» de l'abbé Ferland, "Le Journal de deux voyages apostoliques de $\mathrm{M}^{\mathrm{gr}}$ Plessis» et la biographie de F.-X. Garneau de l'abbé Casgrain. De son côté, Antoine Gérin-Lajoie montre bien avec son Jean Rivard, économiste ce qu'il entend par littérature utile. La revue contribue ainsi d'une certaine manière à freiner l'impulsion qu'elle avait donnée au mouvement littéraire.

De son côté, Taché persiste à publier Les Soirées canadiennes, malgré la perte de ses meilleurs collaborateurs. Heureusement qu'il dispose de «Forestiers et Voyageurs», un manuscrit tout prêt, pour remplir les livraisons de l'année 1863. Bientôt réduit à modeler sa conduite sur celle de ses concurrents, il publie, la même année, la «Lettre de l'abbé Maillard sur les missions micmaques», un document inédit du XVIII ${ }^{\mathrm{e}}$ siècle. L'année suivante, deux récits de voyage, «Souvenir de voyage» de Napoléon Bourassa et «Notes d'un condamné politique» de F.-X. Prieur occupent presque à eux seuls toutes les livraisons de l'année. «Souvenirs d'un voyage en Californie» de Philéas Verchère de Boucherville constitue le plat de résistance pour

29. «Prospectus», Le Foyer canadien (Québec, Bureaux du Foyer canadien, 1863), I: 6.

30. Ibid., 7 . 
l'année 1865. Évidemment à court de collaborateurs, Taché se rabat sur des textes plutôt longs qui lui permettent de tenir le coup sans avoir à solliciter de nouveaux collaborateurs. Sa situation critique l'oblige à renoncer à son dessein originel pour adopter la formule de ses adversaires. Les vieux fonds de manuscrits ne suffisent cependant pas à alimenter deux revues indéfiniment. Aussi doivent-elles bientôt déclarer forfait, et ce sont les Montréalais qui sauront profiter de la leçon pour leur Revue canadienne.

En résumé, affirmons que si le groupe des Soirées canadiennes avaient réellement amorcé un mouvement littéraire d'envergure, il s'est chargé lui-même de le saborder par sa mésentente sur la notion de littérature.

\section{LA REVUE CANADIENNE}

À l'époque où la zizanie divise l'équipe québécoise des Soirées canadiennes, un groupe de lettrés montréalais entrent en lice avec une nouvelle revue. On peut se demander si La Revue canadienne n'est pas l'expression d'un groupe analogue à celui de Québec. De prime abord, la nouvelle revue ne semble pas manifester l'existence d'un groupe de lettrés le moindrement structuré.

$\mathrm{Au}$ contraire, on peut croire qu'elle est plutôt le résultat d'une initiative personnelle. Même si Napoléon Bourassa apparaît comme premier président dans les procès-verbaux, il n'en est pas le véritable initiateur. De retour de son séjour d'études à Rome depuis décembre 1855 , il s'est fait connaître par des conférences sur l'art et par le récit de ses voyages. De plus, il s'est clairement identifié au mouvement ultramontain par sa fréquentation du Cabinet de lecture paroissial et par son élection, en 1862, au comité de direction de l'Union catholique dont il assume la présidence en 1863. Plus esthète et dilettante que chef, Bourassa sert de tête d'affiche à quelques jeunes gens qui ont besoin de la caution d'un aîné pour assurer leur crédibilité. En fait, les fondateurs de la nouvelle revue se recrutent dans les rangs des anciens du collège Sainte-Marie.

Dès leurs premières années d'enseignement, les jésuites ont noyauté une certaine jeunesse au sein de leur Union catholique pour en faire les apôtres laïques de l'ultramontanisme. De ceux-là émergent très tôt Joseph Royal, Cyrille Boucher, Édouard Lefebvre de Bellefeuille et Louis Beaubien, qui constituent une première phalange du journalisme ultramontain. Après la disparition des Mélanges religieux, au cours du grand incendie de 1852, le diocèse de Montréal se cherche un nouvel organe de presse. Bien convaincu de l'efficacité de l'apostolat des médias, Joseph Royal, d'abord rédacteur à La Minerve, fonde 
en 1858, avec Cyrille Boucher, L'Ordre. Union catholique, un journal ultramontain radical. Mais l'achat du périodique par le libéral Plinquet, en 1861, le prive de sa tribune. En 1862, tout en acquérant les droits de L'Écho du Cabinet de lecture paroissial, Joseph Royal rêve d'une revue de plus d'envergure. Certainement instruit par les déboires des Soirées canadiennes, trop centrée sur la littérature, il vise un plus vaste bassin de lecteurs en adoptant la formule de La Revue des Deux Mondes, bien connue des milieux intellectuels. Il entend intéresser tant les scientifiques, les juristes, les économistes, les philosophes, que les littéraires. C'est dans cet esprit qu'il forme son comité de rédaction dont il offre la présidence à Napoléon Bourassa, capable de tenir la chronique des arts et de juger des articles en ce domaine. Malheureusement, Royal ne peut compter en 1864 sur la collaboration de son fidèle compagnon d'armes, Cyrille Boucher, déjà sérieusement malade - il décède en 1865. Mais Édouard Lefebvre de Bellefeuille, un compagnon de la première heure, le soutient toujours. Les deux s'adjoignent un tout jeune homme, Joseph-Alfred-Norbert Provencher, le neveu de $\mathrm{M}^{\mathrm{gr}}$ Provencher, qui vient de succéder à Évariste Gélinas comme rédacteur de La Minerve. Les autres membres du comité de rédaction semblent avoir été recrutés pour leur spécialité: Paul Letondal pour la musique, Adélard-Joseph Boucher pour les questions de numismatique et d'archéologie, Denis-Henri Senécal pour la littérature et la poésie, le $\mathrm{D}^{\mathrm{r}}$ Hector Peltier, professeur à l'école de médecine, pour les questions scientifiques, et $\mathrm{W}$. Tessier, trésorier de la cité de Montréal, pour les questions économiques. Joseph Royal se réserve, avec Lefebvre de Bellefeuille, les questions de droit. De plus, il assume les fonctions de secrétaire-gérant qui lui valent un salaire.

À l'instar des grandes revues, les directeurs se proposent de payer leurs collaborateurs. Ils passent une entente avec l'imprimeur-éditeur, Eusèbe Senécal, à l'effet que les profits dépassant 1400 seront versés au comité de direction pour la rétribution des articles ${ }^{31}$. Au début, on ne sait trop comment évaluer les diverses contributions: par exemple, on alloue un montant forfaitaire de $250 \$$ à Georges Boucher de Boucherville pour son feuilleton Une de perdue, deux de trouvées et un autre de $300 \$$ à Napoléon Bourassa pour Jacques et Marie. Ces cachets, énormes pour l'époque, seront rajustés à la baisse plus tard. Mais ils prouvent qu'on entend bien attribuer une valeur marchande aux travaux de plume. Pour des articles de moindre envergure, les cachets varient entre $6 \$$ et $15 \$$. Toutefois, à sa séance du 23 décembre

31. Ces détails et ceux qui suivent sont tirés des procès-verbaux de la Revue canadienne déposés aux archives de la Bibliothèque municipale de Montréal, collection Gagnon. 
1864 , le comité se ravise: le maximum sera de $12 \$$ et le minimum de $3 \$$.

Cette initiative contribue rapidement au succès de la nouvelle revue. Crémazie feint l'étonnement dans une lettre à l'abbé Casgrain: « [...] mais on cherche vainement dans votre recueil les noms des jeunes écrivains qui faisaient partie du comité de collaboration formé à la naissance du Foyer. Pourquoi toutes ces voix sont-elles muettes ${ }^{32}$ ?» Le poète sait bien que ces jeunes écrivains préfèrent vendre leurs textes plutôt que de les donner quand ils en ont l'occasion. Cette concurrence aura pour effet, à très court terme, de déclasser complètement les revues québécoises. Pour remplir ses pages, $L e$ Foyer canadien en est bientôt réduit à publier en feuilleton $L e$ Fratricide, roman du vicomte Walsh.

La Revue canadienne peut se permettre de pareilles largesses apparemment à cause du succès de sa campagne d'abonnements. En effet, le procès-verbal de la réunion du 10 avril 1864 note un tirage de 1306 exemplaires dont 50 sont réservés aux échanges. Comment expliquer ce succès? Remarquons d'abord que Les Soirées canadiennes, malgré sa prétention au titre de recueil de littérature nationale, n'avait pas réussi à percer le marché montréalais. Dans la liste des abonnés en appendice du premier volume des Soirées, on ne compte que 42 abonnements à Montréal contre 376 à Québec. Ce qui confirme une vocation plutôt régionale. À vrai dire, La Revue canadienne n'entre pas en concurrence avec les revues québécoises, car le marché montréalais demeure virtuellement vierge.

Mais il y a plus: La Revue canadienne respecte l'esprit de ce genre de publication. Au lieu de se limiter à la littérature et à l'histoire, elle s'ouvre aux sciences, au droit, à la religion, à l'économie et à la philosophie. Plusieurs numéros se terminent par la recension de quelques nouveautés et par une chronique sur les événements du mois. Cette variété la met à la portée d'un plus grand nombre de lecteurs. On ne tarde donc pas à faire des comparaisons désavantageuses pour les revues de Québec. Crémazie s'en ouvre à Casgrain: «Avec ce système, Le Foyer n'est plus une revue; c'est tout simplement une série d'ouvrages publiés par livraison [...]. Il est évident que si pendant cinq ou six mois, un abonné ne trouve dans Le Foyer qu'une lecture sans attrait pour lui, il prendra votre recueil en dégoût et ne tardera pas à se désabonner ${ }^{33}$.» En effet les revues québécoises avec le temps sont

32. O. Crémazie à H. R. Casgrain, 29 janvier 1867, H. R. Casgrain, Euvres complètes (Montréal, Beauchemin \& Valois, 1885), II: 365.

33. Ibid., 366. 
devenues des clubs du livre avant la lettre qui comptent sur une clientèle captive pour publier des ouvrages qui autrement resteraient dans les tiroirs.

La Revue canadienne ne laisse pas tomber les littéraires pour autant. Elle se fait même aguichante en publiant dans chaque numéro un feuilleton qui pique la curiosité du lecteur. Une de perdue, deux de trouvées, Jacques et Marie et Nélida ou les Guerres canadiennes gagnent certainement les lecteurs les plus rébarbatifs. Les amateurs de poésie y trouvent également leur compte. Les nombreux récits de voyage captivent les esprits moins rigoureux. Enfin, les chroniques fournissent une information nécessaire à ceux qui veulent se tenir au courant de l'actualité.

La Revue canadienne se démarque aussi de ses concurrentes québécoises par la variété de ses collaborateurs. Dès le 10 février, on note dans le procès-verbal les réponses favorables de collaborateurs éventuels que l'on a sollicités. Ce sont Jean-Baptiste Meilleur, Paul Stevens, Hector Fabre, l'abbé Antonin Nantel, l'abbé Joseph-Sabin Raymond, Hector Langevin, l'abbé O’Donnell, Félix-Gabriel Marchand, Georges Laberge et James McPherson LeMoyne. Plusieurs autres collaborateurs enverront spontanément des textes à la revue. C'est ainsi que le comité de rédaction peut exercer une sélection. À chaque réunion, il rend son verdict en ces termes: pour publication dans le prochain numéro; publication ajournée à plus tard; publication ajournée indéfiniment.

Les jugements qui sont alors portés ne le sont pas uniquement en fonction de la valeur intrinsèque du texte, comme le sommaire de chaque numéro nous porte à le croire. L'orientation ultramontaine de la revue saute aux yeux. Les articles de fond de Joseph-Sabin Raymond couvrent plusieurs livraisons de la revue; par exemple: Destinée providentielle de Rome en six tranches; De l'Église et de l'État également en six tranches. Napoléon Bourassa, qui remémore ses souvenirs de voyage en Italie, familiarise le lecteur avec la Rome pontificale. Les piliers de l'ultramontanisme, comme $\mathrm{M}^{\mathrm{gr}}$ Joseph Desautels et le chanoine Godefroy Lamarche, y ont leurs entrées. Des questions brûlantes, comme le démembrement de la paroisse NotreDame de Montréal, donnent à Lefebvre de Bellefeuille l'occasion de soutenir $\mathrm{M}^{\mathrm{gr}}$ Bourget contre les sulpiciens.

Mais il serait abusif d'affirmer que l'orientation idéologique prime partout et toujours. Les pages de la revue sont largement ouvertes aux divers genres littéraires. La direction semble avoir compté sur les feuilletons pour attirer des lecteurs. Dès le début, elle adopte comme politique de publier dans chaque numéro un épisode d'un roman 
spécialement commandé à un écrivain. Elle consacre des sommes considérables pour acheter les deux premiers manuscrits, soit Une de perdue, deux de trouvées et Jacques et Marie. Mais, en août 1866, la caisse de l'entreprise ne semble plus suffire pour honorer des commandes d'une telle envergure. Le nouveau feuilleton, Charles et Éva de Joseph Marmette, paraît en trois livraisons seulement (décembre 1866, février et mai 1867). Le choix de Nélida ou les Guerres canadiennes de 1812 du Belge Thil-Lorrain paraît révélateur. Pourquoi recourt-on à cet étranger plutôt qu'à un auteur canadien, si ce n'est parce qu'on peut obtenir son texte à meilleur marché? Nélida paraît en effet chez Casterman à Tournai la même année. En juillet 1867, la direction revient à une œuvre locale, Scènes de la guerre d'indépendance du Mexique d'Édouard Lefebvre de Bellefeuille, un membre de l'équipe de rédaction suffisamment argenté pour se passer de cachet.

Les autres feuilletons jusqu'à la fin de la décennie appuient l'hypothèse d'une insuffisance de fonds pour commander des œuvres originales. En avril 1868, la publication d'Anne Séverin de madame Augustus Craven (Pauline de La Ferronnays, 1808-1891) publié à Paris fait croire à une édition pirate. Pendant la dernière année de la décennie, paraissent deux romans d'obscurs auteurs français: Une famille parisienne d'un certain Hypolite [sic] Audeval et Deux épaves de M. Parseval-Deschênes. Pourquoi ces choix plutôt aberrants? Les romanciers canadiens sont-ils tombés en panne? Ou plutôt la revue, voyant ses revenus décroître, n'a-t-elle plus les moyens de jouer le rôle qu'elle s'était d'abord assigné, soit de susciter de nouvelles créations littéraires?

La même politique semble avoir prévalu pour la poésie. Les cachets versés aux poètes devaient stimuler la création. Toutefois, les résultats déçoivent les observateurs contemporains. De janvier 1864 à décembre 1869 , la revue ne publie que 32 poèmes pour un total de 72 livraisons. Nouvelle interrogation: s'agit-il d'une politique éditoriale ou d'un manque de manuscrits? Il semble bien que les poètes n'aient pas répondu à l'appel de la revue comme ils l'auraient dû. Seul Benjamin Sulte aurait profité pleinement de l'hospitalité du périodique. À lui seul, il livre plus du tiers des poèmes. Alfred Garneau, Pamphile Lemay et Eustache Prud'homme soumettent chacun trois poèmes.

La Revue canadienne ne privilégie pas une forme de poésie plutôt qu'une autre. Aussi, ses poètes ne se réclament-ils d'aucune école. Certes, elle se fait fort de publier des participants au concours de poésie de l'Université Laval ${ }^{34}$ et quelques poèmes à saveur patriotique,

34. Achille Fréchette, «Les Martyrs de la foi en Canada», Revue canadienne (septembre 1868): 678 . 
comme «Les Pionniers ${ }^{35}$ » et le «Chant des artisans canadiens ${ }^{36}$ » de Sulte, mais en général, elle accepte les poèmes plutôt pour leur valeur formelle que pour leur valeur idéologique. Malgré une absence de directive, les jeunes poètes qui gravitent autour de La Revue canadienne ont certaines convergences dans leur inspiration. Des poèmes comme «Le Village Huron à Lorette» de Lemay ${ }^{37}$, «La Belle Meunière» de Sulte ${ }^{38}$, «Mon village» de Prudhomme ${ }^{39}$, «La Débâcle du Saint-Laurent» de Lemay ${ }^{40}$ annoncent le régionalisme caractéristique de la poésie canadienne au tournant du siècle. Par ailleurs, d'autres poèmes à saveur nettement intimiste, comme ceux d'Alfred Garneau et un en particulier de Prud'homme ${ }^{41}$, laissent deviner la grande latitude accordée par la rédaction. Aussi ne peut-on considérer cette revue comme une école qui aurait exercé une influence déterminante sur la poésie de l'époque.

Un examen rapide du sommaire de la revue confirme la popularité du récit de voyage. Napoléon Bourassa ${ }^{42}$ et J.-S. Raymond avec ses «Entretiens sur Naples» y tiennent une place de choix. Faucher de Saint-Maurice livre à partir de septembre 1866 son long récit de voyage «De Québec à Mexico», «Aventures et Voyages, La Petrolie» s'inscrivent au sommaire des numéros d'avril, mai, juillet et août 1866. Sous la rubrique «Extraits de notes de voyage», Louis-Rodrigue Masson évoque ses souvenirs de divers lieux célèbres de l'Europe (début novembre 1866). L'abbé L.-J. Huot fait agréer son «Journal de voyage en Europe» pour la livraison d'avril 1868. Ces récits, qu'ils concernent l'Italie, le Mexique ou la France, exaltent en général l'ancien ordre des choses aux dépens de la Révolution. Au contraire des fresques romantiques que brossent Bourassa et Raymond, Faucher de Saint-Maurice inaugure un genre bien personnel de récit de voyage.

Sous la rubrique «Notice bibliographique», La Revue canadienne recense les dernières parutions. Mais dans la majeure partie des cas, il s'agit d'accusés de réception ou encore de brèves analyses de contenu. Rarement ose-t-on porter des jugements. De plus, dans une revue non exclusivement littéraire, on s'intéresse à des ouvrages de toute sorte, allant du droit aux sciences naturelles. La littérature y tient peu d'espace. Au cours des premières années, seuls quelques titres

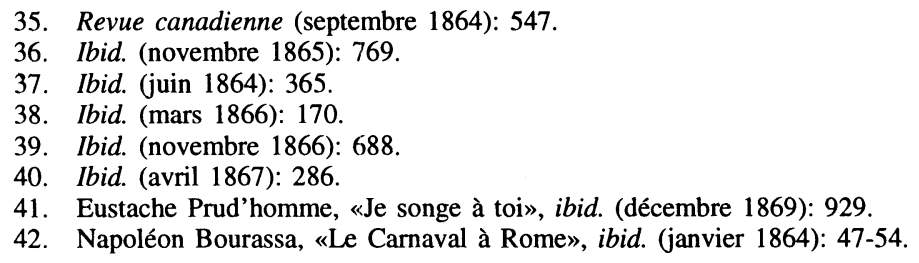


d'œuvres littéraires retiennent l'attention. Édouard Lefebvre de Bellefeuille critique Antoinette de Mirecourt de madame Leprohon, sa tante par alliance. Joseph Royal se contente d'accuser réception des Essais poétiques de Pamphile Lemay. Seule La Grand-Tronciade fait l'objet d'un éreintement de la part de Pamphile Lemay ${ }^{43}$. Curieusement, c'est sous la rubrique «Les Événements du mois» que Siméon Lesage célèbre la parution des Mémoires de Philippe Aubert de Gaspé. Comme l'auteur des Anciens Canadiens jouit déjà de la consécration littéraire, le critique ne porte son attention que sur des points positifs: témoin privilégié de l'ancienne aristocratie, Aubert de Gaspé fait un choix judicieux parmi ses souvenirs. Observateur fin et délicat, il peint ses amis avec la «mémoire du cœur». Le récit lui paraît valoir plus par sa révélation de «mœurs aujourd'hui disparues» que par ses qualités littéraires.

L'histoire jouit d'un traitement privilégié. Avouons que c'est une période faste. La parution des deuxième et troisième tomes de l'Histoire de la colonie française en Canada de l'abbé Michel Faillon mérite plus qu'une simple recension. C'est un éloge en règle que Joseph Royal adresse à l'historien qui révèle les traits historiques confirmant la mission providentielle du peuple canadien-français ${ }^{44}$. La recension de l'Histoire de la Mère Marie de l'Incarnation de l'abbé Casgrain en janvier 1865 jointe, en juillet 1867, à celle du second volume du Cours d'histoire du Canada de l'abbé Ferland consacrent l'hégémonie des prêtres historiens sur le genre.

En plus des grands ouvrages déjà mentionnés, on recense nombre d'histoires secondaires, comme L'Histoire des Abénakis de l'abbé A.J. Maurault (janvier 1867), Histoire des grandes familles françaises du Canada de l'abbé Daniel (mai 1868), la monographie du père Félix Martin intitulée De Montcalm en Canada (août 1868); Haliburton, The Men to the North... (juin 1869); T.-B. Bédard, Histoire de cinquante ans (1791-1840) (décembre 1869). Enfin, on signale les rééditions des écrits de la Nouvelle-France propres à alimenter les recherches historiques.

Évidemment cette critique se contente d'exprimer les consensus de la majorité sans jamais se hasarder à aller à contre-courant. Mais elle n'en affiche pas moins des codes qui prévalent dans le monde littéraire depuis le renouveau catholique.

La Revue canadienne a trouvé une formule beaucoup plus profitable que les revues québécoises, comme le prouve une longévité

43. Ibid. (juillet 1866): 441-442.

44. Ibid. (octobre 1865): 627; (mars 1866): 181; (mai 1866): 313. 
incomparable - elle dure jusqu'en 1922. Mais son rôle dans la vie littéraire n'est pas aussi déterminant qu'on aurait pu l'espérer. En élargissant son champ d'intérêt, elle augmente d'autant ses collaborateurs et ses lecteurs, mais elle dilue son contenu. Toutes les tendances de droite s'y expriment, le comité de rédaction se montrant plus accueillant qu'on aurait pu le prévoir. Au contraire des revues québécoises, cette revue n'est pas d'abord le porte-parole d'un regroupement d'écrivains qui aspirent à donner une impulsion particulière à la littérature. Même si elle dure plus longtemps, La Revue canadienne ne joue pas un rôle comparable à celui des Soirées canadiennes et du Foyer Canadien. Elle fait cependant la preuve qu'avec des fonds plus considérables elle aurait pu stimuler bien davantage la création littéraire, comme elle en avait l'intention à ses débuts.

\section{CONCLUSION}

On peut considérer la période $1840-1870$ comme celle des revues littéraires. Le lectorat marque une certaine fragmentation par comparaison avec la période précédente: le journal ne répond plus à tous les besoins, mais le lectorat littéraire n'est pas encore assez nombreux pour justifier la publication en livre. La revue apparaît donc comme un compromis. Avec ses abonnés, elle compte sur un public captif et s'assure ainsi des tirages suffisants pour rentabiliser la publication. Aussi est-on tenté d'abuser de la formule pour éditer des ouvrages qui autrement ne trouveraient pas preneur. Les lecteurs ne se laissent pas duper et abandonnent les revues qui n'en sont pas. La Revue canadienne survit parce qu'elle respecte vraiment la formule.

Mais ces revues, répertoires ou recueils jouent un autre rôle que le livre ou le journal en ce sens qu'ils sélectionnent les textes en vertu de leur valeur littéraire. De cette façon, ils participent à la création d'un champ qui attire les littéraires qui veulent se mesurer les uns aux autres. Mais ce champ, du moins pendant cette période, manque de compétitivité parce que la notion de littérature demeure encore trop vague. Trop de disciplines cohabitent pour que chacun des acteurs puissent se trouver un vis-à-vis de taille. Aussi la consécration de la valeur s'en ressent-elle. On se demande encore si les textes ont été retenus comme documents ou comme œuvres. 